\title{
Adenocarcinoma of the Lung Mimicking Miliary Tuberculosis
}

\author{
Dawlat Khana ${ }^{a}$ Mohammed Danjuma ${ }^{a} \quad$ Muhammad Umar Saddique ${ }^{a}$ \\ Khaled Abdelfattah Hasan Murshed ${ }^{b}$ Mohamed A. Yassin ${ }^{c}$ \\ aDepartment of Medicine, Hamad General Hospital - Hamad Medical Corporation Qatar, \\ Doha, Qatar; ${ }^{b}$ Department of Pathology, Hamad General Hospital - Hamad Medical \\ Corporation Qatar, Doha, Qatar; ' Department of Medical Oncology-Hematology, Hamad \\ Medical Corporation Qatar, Doha, Qatar
}

\section{Keywords \\ Miliary shadows/pattern · Miliary tuberculosis · Adenocarcinoma - Intrapulmonary metastases.}

\begin{abstract}
Miliary shadows on chest imaging have wide differential diagnoses. The most common etiology is infectious, such as miliary tuberculosis (TB) and histoplasmosis, but miliary shadows can be the presentation of sarcoidosis, pneumoconiosis, and secondary metastasis to the lungs from primary cancers of the thyroid, kidney, and trophoblasts as well as sarcomas. Here we present the case of a 35-year-old Indian male who presented with a 2-month history of dry cough and shortness of breath. Chest imaging showed diffuse bilateral miliary nodules. The initial impression was that of miliary pulmonary TB. Subsequent bronchoscopy with a transbronchial biopsy confirmed the diagnosis of pulmonary mucinous adenocarcinoma with brain metastasis, which is a rare and unusual presentation of primary lung cancer. The tumor was positive for ALK5A4 and PD-L1, and the patient was started on tyrosine kinase inhibitor immunotherapy, with a favorable response.

(C) 2020 The Author(s).

Published by S. Karger AG, Basel
\end{abstract}

\section{Introduction}

Miliary shadows in the lung are seen in a wide variety of conditions. The miliary pattern shows as numerous small nodules (usually 1-3 $\mathrm{mm}$ in diameter) in the lung with sharp margins [1].

The miliary pattern on chest radiography could be a presentation of miliary tuberculosis (TB), histoplasmosis, sarcoidosis, pneumoconiosis, bronchoalveolar carcinoma, or pulmonary 
siderosis. It can also be the sign of secondary metastasis to the lungs from primary cancers of the thyroid, kidney, and trophoblasts as well as some sarcomas [2, 3].

Lung cancer is among the most frequently diagnosed cancers and the world's leading cause of cancer-related death [4]. Lung cancers are classified into small cell lung cancer (SCLC) and non-SCLC (NSCLC). NSCLCs are further divided into three major subtypes: squamous cell carcinoma, adenocarcinoma, and large cell carcinoma. Lung adenocarcinoma is the most common type among nonsmokers and women $[4,5]$.

Lung adenocarcinomas typically arise from the bronchial epithelium or bronchial glandular epithelium. Pathologically, adenocarcinoma may display an acinar, papillary, micropapillary, lepidic, or solid growth pattern, with either mucin or pneumocyte marker expression, and it is usually located peripherally with pleural involvement [6]. Their most frequent radiological demonstration is ground-glass, part-solid, and solid nodules on chest CT scans [7]. Primary lung cancer rarely presents as miliary nodules [8-10]. Here, we present the case of a 35-year-old male nonsmoking patient from a TB endemic area presenting with miliary mottling on chest X-ray and diagnosed as having adenocarcinoma of the lung.

\section{Case Presentation}

A 35-year-old male patient from a TB endemic area presented to the emergency department with a 2-month history of dry cough, shortness of breath, and right-sided chest pain. Hehad two episodes of hemoptysis with small amounts of blood 1 week before presenting to the hospital. He had no history of fever or night sweats and had lost around 2-3 kg over the preceding 2 months.

He had no history of close TB contact, incarceration, or recent traveling. He is a nonsmoker with no significant past medical and surgical history and works as a carpenter. There was no history of high-risk sexual behavior and no history of drug addiction.

His vital signs and physical examination results were benign except for a dull percussion note in the right lower chest and decreased air entry on auscultation. A laboratory examination revealed a hemoglobin level of $14 \mathrm{~g} / \mathrm{dL}$ (reference range 13.0-17.0) and a total leukocyte count of $9,300 / \mathrm{mm}^{3}$ (reference range 4.0-10.0), but normal differential counts. His liver and renal function test results were within normal limits. The $\mathrm{C}$-reactive protein level was $22 \mathrm{mg} / \mathrm{L}$ (reference range 0-5). A chest X-ray showed extensive bilateral pulmonary infiltrates with a miliary pattern, and right-sided pleural effusion (Fig. 1). The patient was initially placed on airborne precautions. The following tests done to narrow down the differential diagnoses were all negative: QuantiFERON-TB Gold test, sputum acid-fast bacilli smear (two samples), tuberculin skin test, human immunodeficiency virus antibody test, and hypersensitivity pneumonitis screen.

A CT scan of the chest confirmed multiple miliary nodular infiltrates throughout both lungs, mediastinal lymphadenopathy, and a right hilar soft tissue lesion, as well as multiple dense dorsal spinal lesions and abdominal retroperitoneal lymphadenopathy (Fig. 2, 3). Whole-body PET further confirmed a primary tumor in the right lung with bilateral extensive small nodular pulmonary metastases; bilateral hilar, multiple mediastinal, left lower neck, and upper retroperitoneal and para-aortic lymph node metastases; and multiple sclerotic bone metastases. Head MRI revealed multiple (about 5) foci of abnormal enhancement, scattered in both cerebral hemispheres but mainly on the left side, likely metastatic.

Fiberoptic flexible video bronchoscopy was performed, revealing unremarkable cords, trachea, and carina and left lung. The right side of the lung showed nodular infiltration starting from the right main bronchus extending into the right bronchus intermedius, causing complete occlusion of the right lower lobe apical segment. Bronchioalveolar lavage, bron- 
Fig. 1. Chest X-ray. Posterior-anterior view showing bilateral miliary shadows (red arrows) and right-sided pleural effusion (blue arrow).

Fig. 2. Chest CT scan showing bilateral miliary nodular infiltrates (red arrows).

Fig. 3. Chest CT scan showing multiple bilateral miliary nodules (red arrows).

Fig. 4. Transbronchial biopsy depicting the tumor with an acinar growth pattern. H\&E. $\times 200$.
1
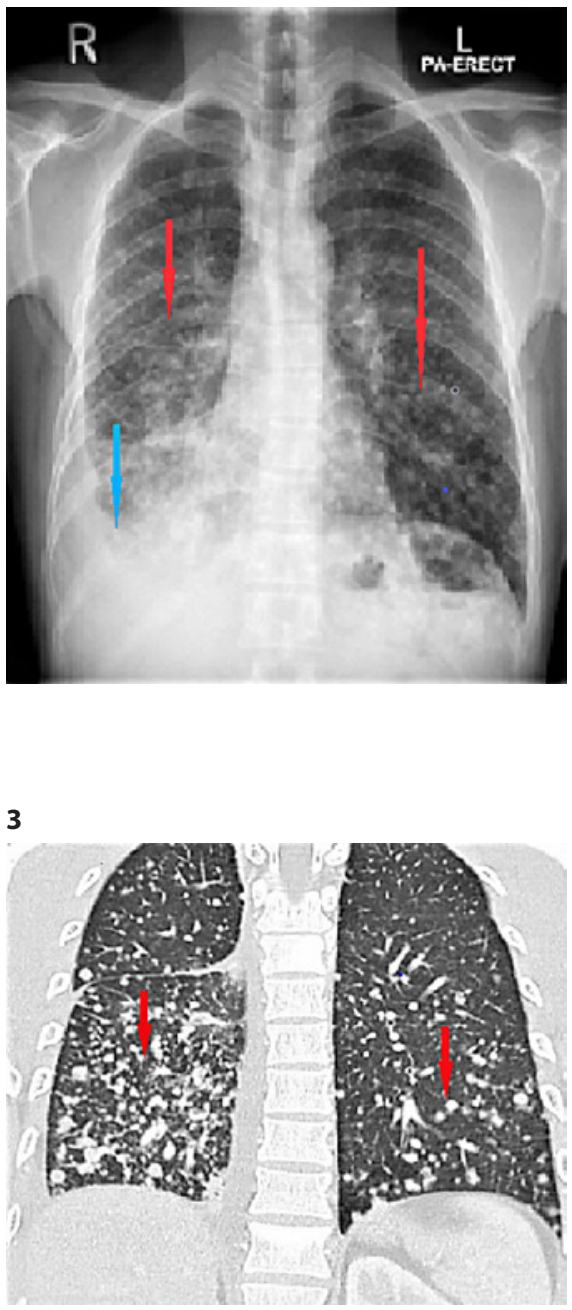

5

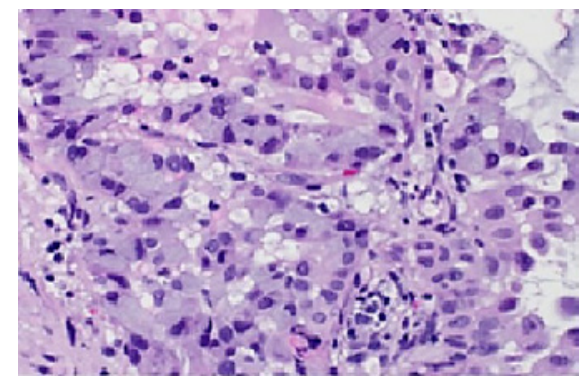

2

4
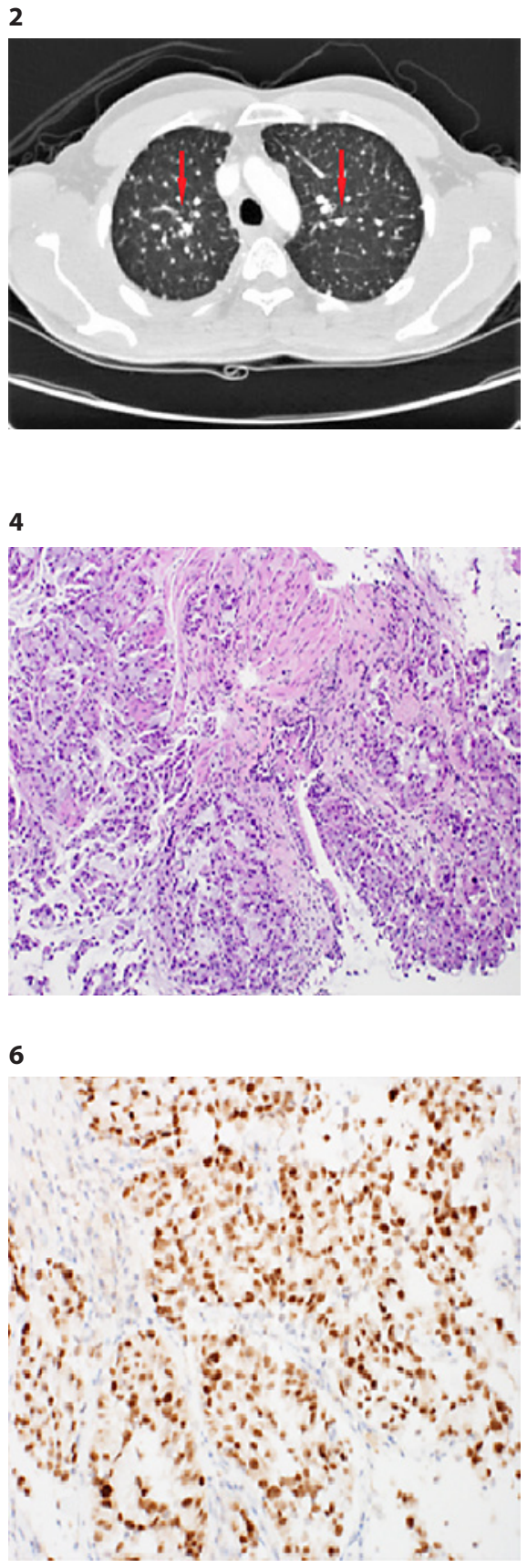

Fig. 5. Tumor cells showing cellular atypia with prominent nuclei and abundant intracytoplasmic mucin. H\&E. $\times 400$.

Fig. 6. Tumor cells demonstrating diffuse strong nuclear staining for TTF-1. chial brushings, and fluoroscopy-guided transbronchial biopsies of the right-sided mass were conducted.

The bronchioalveolar lavage was negative for malignant cells, and histopathology showed pulmonary mucinous adenocarcinoma (Fig. 4-6). Immunohistochemical analysis revealed that the tumor cells were positive for napsin A (Fig. 7) and anaplastic lymphoma kinase 
Fig. 7. Diffuse strong cytoplasmic staining for napsin A.

Fig. 8. Diffuse strong cytoplasmic staining for ALK-1.

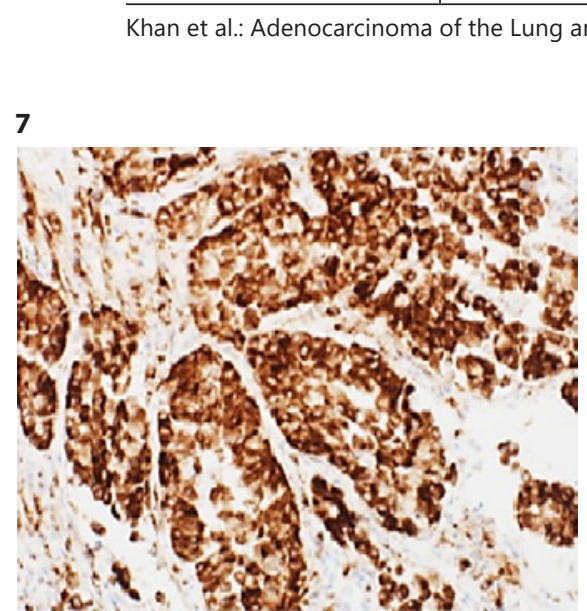

8

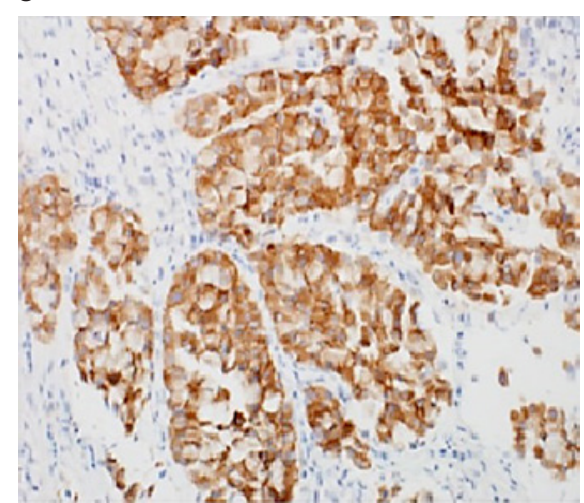

Fig. 9. Moderate-tostrong membranous staining for PD-L1.

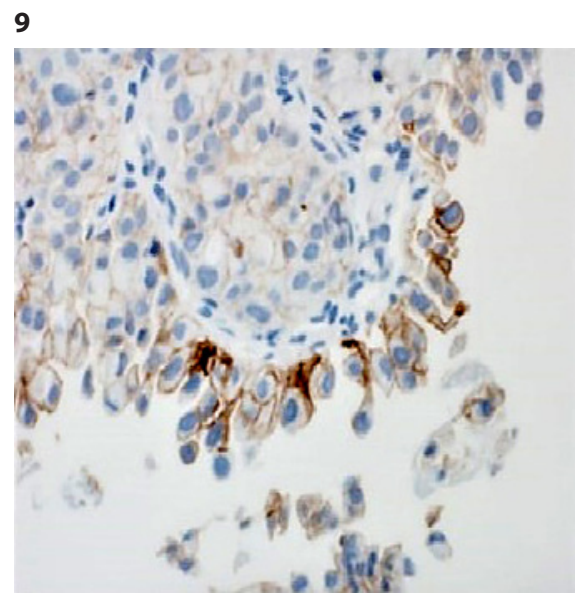

(ALK5A4) (Fig. 8) and strongly positive for programmed cell death ligand 1 (PD-L1) (Fig. 9). EGFR mutation was negative.

The patient was referred to an oncologist and was started on tyrosine kinase inhibitorbased therapy, initially crizotinib, and then switched to alectinib with a favorable response to treatment. PET CT done after 4 months showed remarkable resolution of the hypermetabolic nodule in the lower lobe of the right lung, as well as resolution of the right pleural effusion and the lymphadenopathy in the abdomen and retroperitoneum. Metabolic resolution of the hypermetabolic bone lesions was observed, and there were no new lesions. A repeat head MRI demonstrated a favorable response to treatment. The patient is still under active treatment and tolerating the treatment well.

\section{Discussion}

Lung cancer is among the most frequently diagnosed cancers and the world's leading cause of cancer-related death [4]. The peak-incidence age for lung cancer is between 55 and 65 years, and smoking is a well-established risk factor: active and passive smokers have a 13 -fold and 1.5-fold higher risk than nonsmokers, respectively [11]. Lung cancers are classified into SCLC and NSCLC. NSCLCs are further divided into three major subtypes: squamous cell carcinoma, adenocarcinoma, and large cell carcinoma [4, 5]. Squamous cell carcinoma and SCLC quite often present as a central mass [12]. 
Lung adenocarcinomas typically arise from the bronchial epithelium or bronchial glandular epithelium, and they are the most common type among nonsmokers and women $[4,5]$. Pathologically, adenocarcinoma may display an acinar, papillary, micropapillary, lepidic, or solid growth pattern, with either mucin or pneumocyte marker expression, and it is usually located peripherally with pleural involvement [6]. The most frequent radiological demonstration is ground-glass, part-solid, and solid nodules on chest CT scans [7]. Primary lung cancer rarely presents as miliary nodules [8-10].

Miliary shadows in the lung are seen in a wide variety of conditions. The miliary pattern shows as numerous small nodules (usually 1-3 $\mathrm{mm}$ in diameter) in the lung that usually have sharp margins [1]. The miliary pattern on chest radiography could be a presentation of miliary TB, histoplasmosis, sarcoidosis, pneumoconiosis, bronchoalveolar carcinoma, or pulmonary siderosis. It can also be the sign of secondary metastasis to the lungs from primary cancers of the thyroid, kidney, and trophoblasts as well as some sarcomas [2, 3]. High-resolution CT can help to narrow down the differential diagnoses by distributing the micronodules into centrilobular, perilymphatic, and random patterns. Infectious bronchiolitis commonly presents a centrilobular pattern and sarcoidosis a perilymphatic pattern, whereas a random pattern is suggestive of hematogenous metastases, as in our patient (Fig. 3) [12].

A thorough review of the literature revealed a few similar cases reported as miliary primary lung carcinoma [2]. The largest case series is reported from Japan, in which 5 such patients were included [3]. That report concluded that the prevalence of the miliary phenotype is $<1 \%$ among Japanese patients and that they had a poor prognosis.

Adenocarcinoma is the most common type of lung cancer among nonsmokers and women $[4,5]$. In TB endemic areas, it is always tempting to treat patients presenting with miliary shadows on chest imaging as miliary TB cases [13]. The presentation of adenocarcinoma of the lung as miliary shadows is rare, but it has a poor prognosis if its diagnosis is delayed. Immunotherapies with tyrosine kinase inhibitors and anaplastic lymphoma kinase fusion inhibitors have elicited favorable treatment responses and prolonged overall survival, as in our case [11, 12].

\section{Conclusions}

Miliary shadows on chest imaging could be a presentation of common conditions such as miliary TB, fungal infections, sarcoidosis, and occupational lung disease. It can also be a manifestation of hematogenous metastasis to the lungs from thyroid and renal cancers, sarcomas, etc. Adenocarcinoma of the lung, although rarely presenting as a miliary pattern due to the intrapulmonary nature of its metastases, nevertheless could be missed if no tissue biopsy is taken and patients are treated empirically in TB endemic areas based on a radiological diagnosis of miliary TB. This can lead to a delay in diagnosis and in the initiation of effective tyrosine kinase inhibitor-based treatment and will lead to poor outcomes. We conclude that adenocarcinoma of the lung may be considered a rare but sinister differential diagnosis of miliary shadows on chest imaging. It has a favorable response to tyrosine kinase inhibitorbased therapy even with brain metastasis.

\section{Acknowledgments}

All authors acknowledge the support of the Department of Pathology, Hamad General Hospital Qatar, for providing the histopathology pictures, and the Department of Medical Education, Hamad Medical Corporation Qatar, for proving the basic medical information on the patient. 
Khan et al.: Adenocarcinoma of the Lung and Miliary Shadows

\section{Statement of Ethics}

Consent was obtained from all participants in this study. The Institutional Review Board of the Medical Research Council, Hamad Medical Corporation Qatar, reviewed the protocol and approved it under the condition that no personal identifying information should be mentioned within the publication.

\section{Disclosure Statement}

All authors declare no potential conflicts of interest related to the publication of this case report.

\section{Funding Sources}

The Qatar National Library (QNL) Doha, Qatar, sponsored the publication charges.

\section{Author Contributions}

D.K. conceived and designed the idea, literature review, data collection and radiology figures, wrote the manuscript, and overall organized the case report; M.D. contributed to the literature review and helped in manuscript writing; M.U.S. contributed to data collection and the radiology figures and helped in manuscript writing; K.A.H.M. contributed to the pathology section of the case report and the related literature review; M.A.Y. supervised the project and proof-reading of the manuscript.

\section{References}

1 Njeh H, Abid H, Bradaii M, Msaaed S, Ben Mahfoudh K. Miliary disease of the lung: what is your diagnosis? Eur Respir J. 2013;42(Suppl 57):P2260.

2 Eisenberg RL. Clinical imaging: an atlas of differential diagnoses. 2nd ed. Gaithersburg, MD: Aspen Publishers; 1992. p. 56-9.

3 Koutsopoulos AV, Mitrouska I, Dambaki KI, Voloudaki A, Vardaki E, Haniotis V, et al. Is a miliary chest pattern always indicative of tuberculosis or malignancy? Respiration. 2006;73(3):379-81.

4 Torre LA, Bray F, Siegel RL, Ferlay J, Lortet-Tieulent J, Jemal A. Global cancer statistics, 2012. CA Cancer J Clin. 2015;65(2):87-108.

5 Couraud S, Zalcman G, Milleron B, Morin F, Souquet PJ. Lung cancer in never smokers - a review. Eur J Cancer. 2012;48(9):1299-311.

6 Pillai S, Khan A, Khan S. Adenocarcinoma of the lung presenting with intrapulmonary miliary metastasis. Cureus. 2019;11(8):e5430.

7 Diederich S, Wormanns D, Semik M, Thomas M, Lenzen H, Roos N, et al. Screening for early lung cancer with low-dose spiral CT: prevalence in 817 asymptomatic smokers. Radiology. 2002;222(3):773-81.

8 Henschke CI, McCauley DI, Yankelevitz DF, Naidich DP, McGuinness G, Miettinen OS, et al. Early Lung Cancer Action Project: overall design and findings from baseline screening. Lancet. 1999;354(9173):99-105.

9 Chan A, Devanand A, Low SY, Koh MS. Radial endobronchial ultrasound in diagnosing peripheral lung lesions in a high tuberculosis setting. BMC Pulm Med. 2015;15:90.

10 Fachinger P, Tini GM, Grobholz R, Gambazzi F, Fankhauser H, Irani S. Pulmonary tularaemia: all that looks like cancer is not necessarily cancer - case report of four consecutive cases. BMC Pulm Med. 2015;15:27.

11 Jayaram Subhashchandra B, Ismailkhan M, Chikkaveeraiah Shashidhar K, Gopalakrishna Narahari M. A rare case of non-small cell carcinoma of lung presenting as miliary mottling. Iran J Med Sci. 2013;38(1):65-8.

12 Kimmig L, Bueno J. Miliary nodules: not always tuberculosis. Ann Am Thorac Soc. 2017;14(12):1858-60.

13 Prajapat DK, Khanna A, Talwar D. Adenocarcinoma of the lung masquerading as miliary mottling: a rare presentation. Egypt J Intern Med. 2015;27(3):118-20. 